\title{
Stability Analysis of Roughness Array Wake in a High-Speed Boundary Layer
}

\author{
Meelan Choudhari ${ }^{\dagger}$, Fei Li ${ }^{*}$ \\ NASA Langley Research Center, Hampton, VA 23681 \\ and \\ Jack Edwards \\ North Carolina State University, Raleigh, NC 27695
}

\begin{abstract}
Computations are performed to examine the effects of both an isolated and spanwise periodic array of trip elements on a high-speed laminar boundary layer, so as to identify the potential physical mechanisms underlying an earlier transition to turbulence as a result of the trip(s). In the context of a 0.333 scale model of the Hyper- $X$ forebody configuration, the time accurate solution for an array of ramp shaped trips asymptotes to a stationary field at large times, indicating the likely absence of a strong absolute instability in the mildly separated flow due to the trips. A prominent feature of the wake flow behind the trip array corresponds to streamwise streaks that are further amplified in passing through the compression corner. Stability analysis of the streaks using a spatial, 2D eigenvalue approach reveals the potential for a strong convective instability that might explain the earlier onset of turbulence within the array wake. The dominant modes of streak instability are primarily sustained by the spanwise gradients associated with the streaks and lead to integrated logarithmic amplification factors ( $\mathrm{N}$ factors) approaching 7 over the first ramp of the scaled Hyper-X forebody, and substantially higher over the second ramp. Additional computations are presented to shed further light on the effects of both trip geometry and the presence of a compression corner on the evolution of the streaks.
\end{abstract}

\section{INTRODUCTION}

Surface roughness is known to have a substantial impact on the aerothermodynamic predictions for a hypersonic vehicle, regardless of the state of the boundary layer. When the incoming boundary-layer flow is laminar, the presence of 3D surface roughness tends to accelerate the laminar-turbulent transition process, which can result in a dramatic increase in both drag and aerodynamic heating of the vehicle. In scramjet applications, however, artificial roughness elements are often employed for intentional tripping of the boundary layer on the forebody of the vehicle, in order to prevent engine unstart and to minimize the flow non-uniformities at the entrance to the combustor inlet.

Despite the common presence of surface roughness and its impact on the design of a hypersonic vehicle, the physical mechanisms underlying the phenomenon of roughness-induced transition are far from being understood at this time. (See Refs. [1-3] for recent reviews of the previous work on this topic.) To rectify this deficiency, an intensive research effort has been initiated under NASA's Fundamental Aeronautics Program (Hypersonics Project) as well as space exploration program, and by the United States Air Force. This broad set of efforts encompasses wind tunnel experiments in conventional [4, 5] and quiet flow facilities [6,7], flight experiments [8-12], and fundamental computational activities [13-15]. Resulting measurements and simulation databases should yield a wealth of information concerning the effects of surface roughness on boundary-layer transition (BLT) at supersonic and hypersonic Mach numbers; however, complementary analysis pertaining to the stability characteristics of

\footnotetext{
$\dagger$, * Aerospace Technologist, Computational AeroSciences Branch, M.S. 128.

* Professor, Department of Mechanical and Aerospace Engineering.
} 
the modified boundary-layer flow is equally crucial to fully understand the physical mechanisms underlying the roughness effects on BLT, as well as to translate that knowledge base into reduced order predictive models. Providing such analysis (including the development and/or enhancement of pertinent tools) forms the focus of the work reported in this paper.

As discussed below, there are multiple ways in which a discrete 3D roughness element could influence the laminar-turbulent transition in a hypersonic boundary layer, depending on the size and shape of the roughness element. Empirical observations [16] suggest that given a fixed flow environment the transition front begins to move upstream relative to that over a smooth surface when the roughness height exceeds a critical value. As the height parameter is increased beyond this critical value, the transition location continues to shift upstream until asymptoting to a location in the near wake of the roughness element (or, in certain cases, a finite distance downstream of the roughness element [7]) at what is known as the effective trip height. Both the critical and effective values of the height parameter are influenced by the shape of the roughness element and the external disturbance environment [7].

From the standpoint of hydrodynamic instability paradigm, the threshold behavior at small roughness heights seems difficult to explain and could simply be reflective of a nonzero, but immeasurably weak influence on transition when the roughness height parameter tends to zero. On the other hand, the upstream shift in transition onset location for roughness heights exceeding the critical value could, in principle, be attributed to a finite (and cumulative) destabilizing influence of the roughness element on the boundary layer flow in its wake, which remains convectively unstable for the most part. Of course, as the roughness height continues to increase towards the effective value, the accompanying increase in flow reversal near the roughness element could introduce sufficiently strong absolute instability (similar to the low-speed flows [17]), such that the resulting onset of spontaneous unsteadiness plays an important role during the ensuing transition process. However, due to the stabilizing effect of compressibility on free shear flows, the vortex shedding mechanism may weaken substantially with increasing Mach number and, therefore, the dominant effect of discrete surface roughness on BLT might well be as a modifier of convective instabilities. At any rate, as the streamwise scale of flow evolution becomes shorter with an increasing roughness height, the effects of flow non-parallelism and other flow details in the nearfield of the roughness element may also become increasingly important, introducing additional complexity into the stability analysis at the larger heights. It should be noted that surface roughness may also influence transition by serving as an additional catalyst for receptivity to the external disturbance environment and, also, by scattering any instability waves in the incoming boundary layer. However, the latter two roles are perhaps less significant in the context of boundary-layer tripping at high speeds and will not be explored in the present paper.

An alternative (or additional) explanation for roughness effects on transition may be related to the transient or non-modal growth [18] of roughness-induced disturbances. Certainly, the successful correlation [19] between roughness-induced subcritical transition on blunt nose tips and the optimal, nonmodal amplification factors associated with the (unperturbed) boundary-layer flow provides indirect evidence to support the role of transient growth during roughness-induced BLT, especially in the context of distributed surface roughness.

This paper targets the mean-flow modification due to a spanwise periodic array of roughness (i.e., trip) elements and the stability analysis of the resulting, spanwise modulated boundary-layer flow within the wake of this array. The 0.333 scale Hyper-X model as tested by Berry et al. [4] during trip development for the $\mathrm{X}-43 \mathrm{~A}$ flight configurations is used as the primary test case to assess the relevance of the hydrodynamic instability paradigm to the prediction of BLT in the presence of localized surface roughness and, hence, to garner additional insights into the transition mechanisms responsible for the tripping action. However, effects of roughness elements on a flat plate boundary layer are also 
considered, so as to discern any major differences from trip induced disturbances on the forebody of a hypersonic airbreathing vehicle.

\section{FLOW CONDITIONS AND TRIP GEOMETRY}

The present computations model the Mach 6 flow configuration from the wind tunnel experiments in Ref. 4] at a nominal unit Reynolds number of $\mathrm{Re}=2.2 \times 10^{6} / \mathrm{ft}$. The 0.333 scale model of the Hyper-X forebody tested in these experiments is 28 inches long, with the trip array mounted at 7.4 inches from the leading edge. The model geometry consists of a circular leading edge with a radius of 0.01 inches, followed by three flat ramps providing discrete, sequential, non-isentropic flow compressions ahead of the engine. At the nominal angle of attack equal to 2 degrees, the local compression angles for the three ramps correspond to 4.5, 5.5, and 3 degrees, respectively. The first compression corner (between ramps 1 and 2) is located at 12.4 inches, and the second corner at 17.7 inches. Of the various trip configurations tested in the experiment, trip configuration $2 \mathrm{c}$ was identified as providing the best combination of tripping effectiveness and reduced axial vorticity within the turbulent flow approaching the inlet (the latter being important to reduce the non-uniform heating on the closed cowl [4]). The results presented in Sections III and IV correspond to a trip spacing of $\delta=0.081$ inches and a maximum height of $\mathrm{h}=0.060$ inches. These dimensions were derived from Fig. 3d from Ref. 4 (reproduced below as Fig. 1) in conjunction with the reported value of $\delta$ from Table III in that reference. However, we subsequently learned that the trips used during the Mach 6 experiment were actually sized and built using the Mach 10 predictions of the boundary layer height (i.e., a trip spacing of $\delta=0.12$ inches, and not the corresponding prediction in Table III for the Mach 6 configuration as used herein). Thus, no direct comparisons with the measurements in Ref. [4] will be feasible at this stage.
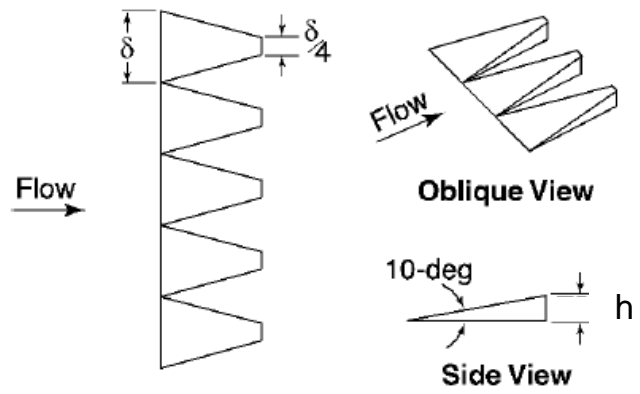

Figure 1. Schematic of trip configuration $2 \mathrm{c}$ (reproduced from ref. [4]). Here, $\delta$ denotes the array spacing and $\mathrm{h}$ represents the maximum height of a trip element.

We note, however, that the existing wind tunnel data for the 0.333-scale Hyper-X configuration includes global heating maps for both passive and active trips, along with oil-flow visualizations along the model surface and off-surface Schlieren images in certain cases.

\section{MEAN FLOW}

To obtain the mean-flow solution in the presence of the trip array, the compressible Navier-Stokes equations are solved using local time stepping in conjunction with an implicit technique. The Piecewise Parabolic Method [20] is used to extend the first-order LDFSS upwind scheme to $4^{\text {th }}$ order for advection terms in smooth regions of the flow. The immersed boundary (IB) technique as implemented by Choi et al. [21] and Edwards [22] is used to simulate the effects of the trip array on the surrounding flow.

We choose to focus our attention on a narrow spanwise region involving a single trip near the symmetry plane of the Hyper-X configuration, and to neglect any large-scale 3D effects (including nonzero crossflow velocities) associated with the finite-span model. Whereas the neglect of finite-span effects is 
essential to make the computation practical, the center-plane flow on the experimental model was most likely influenced by the low aspect ratio of the Hyper-X vehicle geometry.

Consistent with the approximation in the preceding paragraph, a 2D computation was performed to provide an inflow condition for the locally 3D calculation over a single spanwise wavelength of the trip array. The upstream computational boundary for the 3D calculation was located approximately 4 inches downstream of the vehicle leading edge. Previous computations of 2D flows near isolated compression corners have been reported in refs. [23-25]. Compared to the 2D grid, the 3D mesh has a substantially higher streamwise grid density in the vicinity of the trip array, in order to resolve the stronger mean-flow gradients in that region. Due to an earlier misinterpretation of the trip location cited in [4], the trip location in the computation is slightly upstream (by 0.2 ") of that in the experiment. Having confirmed the spanwise symmetry of the flow field during an initial set of computations, the spanwise extent of the computational domain was restricted to one half of the trip spacing, with symmetry conditions imposed along the lateral boundaries. This choice of restricted spanwise domain is supported by the flow visualizations in [4] and [6], which do not reveal any strong subharmonics of the fundamental array spacing. The total number of cells included in the 3D immersed boundary mesh is approximately 12.3 million.

Additional 3D computations on somewhat coarser grids were used to determine an appropriate inflow location for the above 3D computation, as well as to assess the effects of spanwise resolution (varying from 21 points per spanwise wavelength to 81 points per wavelength) on the stationary flow structures within the roughness wake. Time accurate computations performed on the coarser grid provided independent support for the assumption that the flow in the vicinity of the trips will reach a stationary state in the absence of any continuous forcing. Viscous-inviscid interactive hypersonic flows involving flow reversal are known to require relatively larger number of time steps in order to converge to their steady state behavior [26-27]. Accordingly, the computations were continued even after the L2 residual had flattened out around $\mathrm{O}\left(10^{-13}\right)$, so as to ensure that the separated regions were no longer evolving in the eyeball norm.

Finally, as a check on the immersed boundary methodology, additional solutions were obtained on a sequence of body-fitted grids. The finest body fitted grid (denoted henceforth as BF-F) had a cell count of nearly 39 million and the remaining grids were obtained by discarding every other point in the streamwise (BF-Cx), wall-normal (BF-Cy) or both (BF-Cxy) directions.

The presence of the ramp trips leads to a slight region of flow reversal behind the tail of the trip element. Furthermore, the spanwise varying boundary-layer displacement due to the trip elements results in the formation of strong streamwise streaks in the wake of the trip array (Fig. 2). The velocity contours at several streamwise stations reveal that the streaks would persist for long distances over the forebody surface if the boundary-layer flow were to remain laminar throughout that region. Despite the increased momentum transfer associated with these streaks, the modified boundary layer flow does indicate a finite region of flow reversal near the first compression corner, particularly in spanwise regions of upwelling within the boundary layer, as indicated by the corrugated isosurface of the u-velocity component as plotted in Fig. 2.

The presence of longitudinal streaks behind the trip array was also noted in the experiments by Berry et al. [4]. While the streaks induced by the ramp trips appeared to be considerably weak (especially in comparison with those behind diamond shaped trips), we note that the experimental data analysis based on the phosphor thermography system tends to smear out the observations of these streaks as a result of lateral conduction. Recent measurements by Borg et al. [6] also reveal a clear presence of boundary layer streaks for a similar trip configuration on the X-51A model, which was tested under both quiet and noisy 
conditions. The surface heat flux distribution plotted in Fig. 3 appears similar to what might be inferred from the laminar portion of the TSP measurements in ref. [6] under quiet flow conditions.

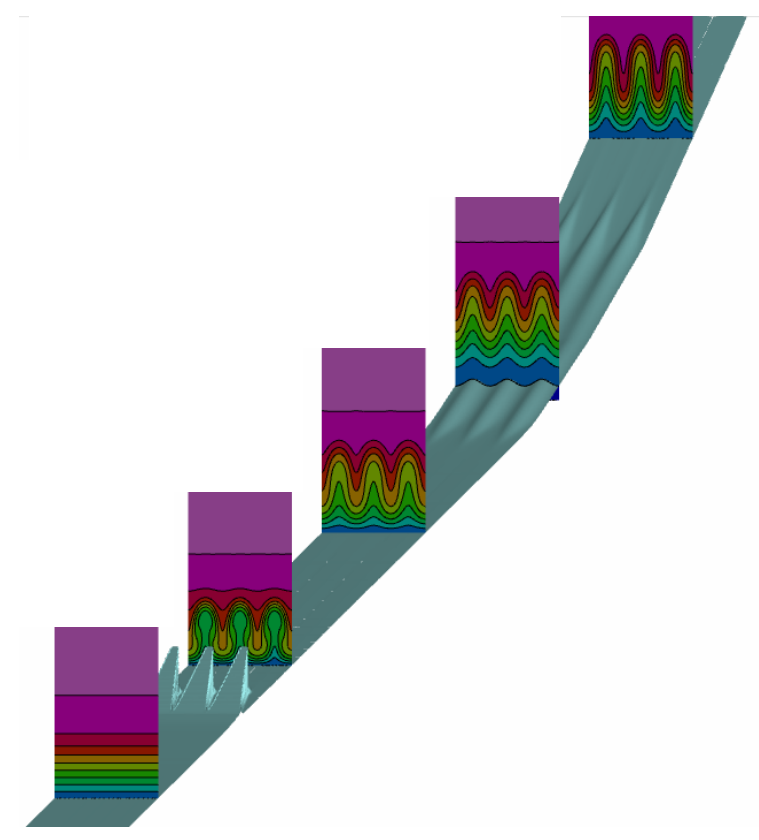

Figure 2. Streamwise streaks produced by trip array (visualized via u-velocity contours at $x=6 "$, 8 ", 10", 12", and 14", respectively; flow is from bottom left to upper right and, for visual clarity, the crossflow coordinates $y$ - $z$ have been magnified relative to the $x$ coordintate). The underlying light blue surface corresponds to the isosurface of streamwise velocity for $\mathrm{u}=1 \mathrm{~m} / \mathrm{sec}$.

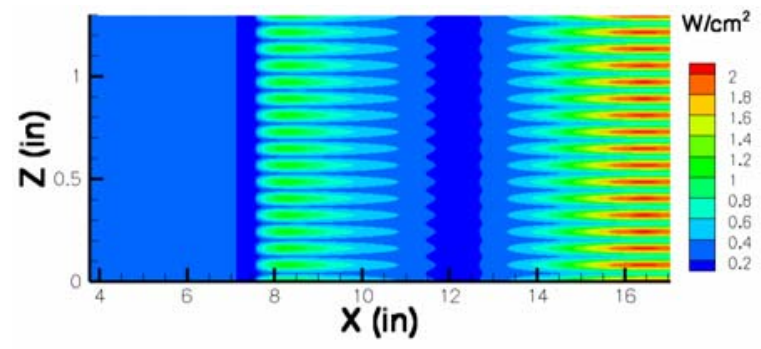

Figure 3. Predicted surface heat flux distribution (excluding the trip region, which is indicated by the dark blue band between 7 and 8 inches).

The computed distribution of surface heat flux along selected spanwise planes across a half-span of the trip width is shown in Fig. 4. The $\mathrm{z} / \delta=0$ and $\mathrm{z} / \delta=0.5$ planes correspond, respectively, to the peak and valley of the local heating behind a trip. The predicted laminar heat transfer to the model surface increases rapidly behind the compression corner $(x / \mathrm{L} \approx 0.26)$, consistent with analogous measurements on the X-51 forebody configuration in a quiet tunnel [6] wherein the flow remained laminar for a finite distance downstream of the compression corner. The measured centerline heating profiles for various roughness heights as reported in ref. [4] for the Hyper-X configuration are shown in Fig. 5. The predicted peak heating due to the laminar streaks is seen to be comparable to the fully turbulent values reported in [4]. However, unlike the purely laminar predictions in Fig. 4, the measured heating for $\mathrm{h}=0.060$ " (and $\delta=$ 0.12 inches as described in Section II) does not involve the pronounced reduction in surface heat transfer just upstream of the first compression corner, suggesting that the onset of transition in the experiment must have been ahead of this corner. 


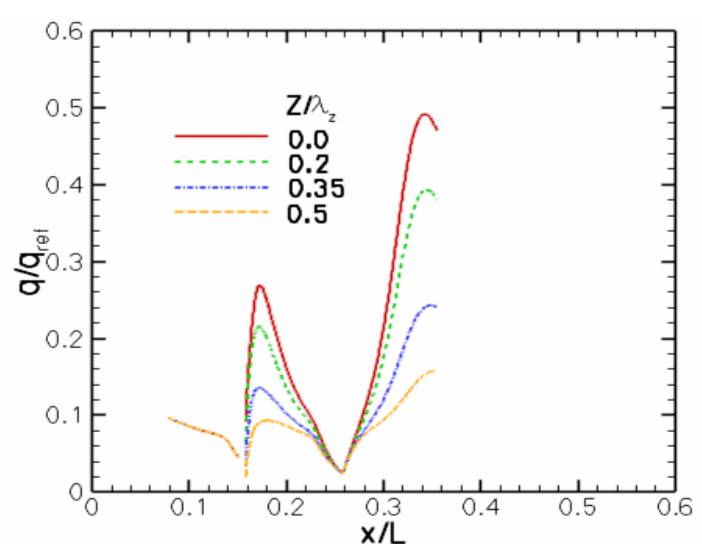

Figure 4. Predicted heat flux distribution at selected spanwise planes. (The streamwise extent of the trip array has been excluded from the plot. The abscissa has been normalized by the reference length of vehicle at the model scale, $\mathrm{L}=48$ ", and the ordinate has been normalized to match the predicted heating at $x / \mathrm{L}=0.1$ with the corresponding laminar value in Fig. 5.)

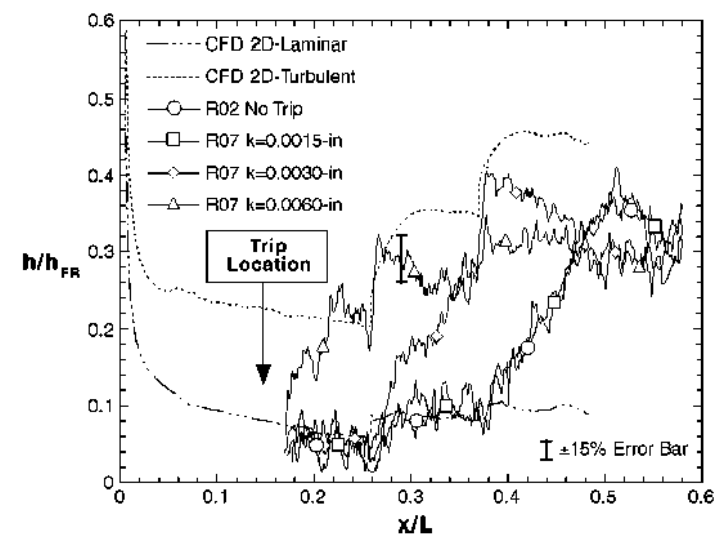

Figure 5. Effect of trip height for Trip 2c on centerline heating profile at Mach 6, $\alpha=2 \mathrm{deg}$, and $\mathrm{Re}=2.2 \times 10^{6} / \mathrm{ft}$ (from ref. [4]).

Fig. 6(a) displays the peak and root mean square (r.m.s.) amplitudes of spanwise variations in the surface heat flux, plotted as functions of the coordinate $x$ along the free-stream direction. The spanwise averaged heat-flux distribution is also included in the plot for comparison purposes. The corresponding amplitudes of the streamwise velocity perturbation associated with the streaks, which are expected to play an important role in determining the instability characteristics of the streaks, are shown in Fig. 6(b). Roughly speaking, the amplitudes of the surface heat-flux modulation track the velocity amplitudes, except that the growth in velocity amplitudes following the initial region of decay behind the trip array is found to lead the onset of increased heat transfer behind the compression corner. Streak amplitudes derived from body-fitted grids of finer to coarser resolutions are also included in Fig. 6(b). The relatively small differences between the various predictions provide confidence that the implementation of the immersed boundary algorithm is correct and the spatial grid is adequate to capture the generation and subsequent evolution of the streaks.

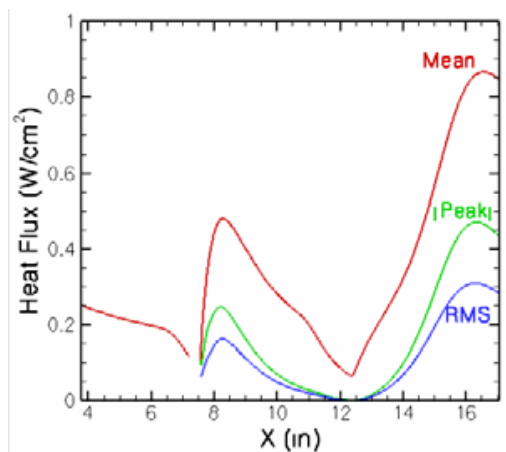

6(a). Amplitudes of surface heat-flux variations, along with spanwise averaged heat transfer

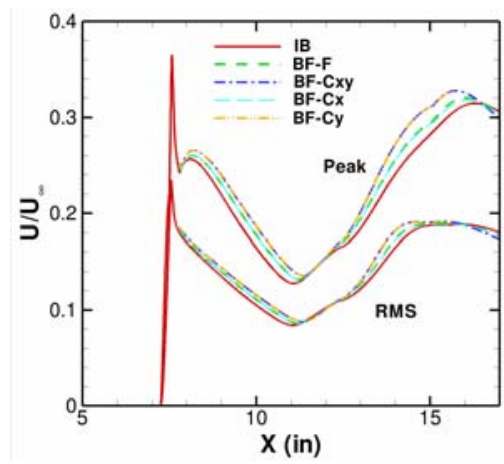

6(b). Amplitudes of tangential velocity perturbation associated with the streaks

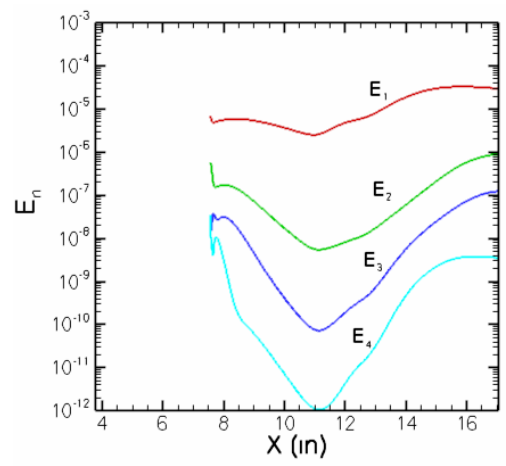

Figure 7. Streamwise evolution of modal energy in dominant Fourier components.

Figure 6. Streamwise evolution of streak amplitudes 
Streamwise evolution of profile-integrated (modal) kinetic energy $\mathrm{E}_{\mathrm{m}}$ for the Fourier modes with spanwise wavelengths of $\lambda=\delta / \mathrm{m}(\mathrm{m}=1-3)$ is shown in Fig. 7. For the 0.060 " trip height considered herein, each of these modes displays a modest growth in energy behind the compression corner (as might have been expected from the overall velocity amplitudes in Fig. 6(b)). The progressive increase in energy growth rate as a function of the harmonic index m suggests that the growth of higher Fourier modes is primarily driven by the nonlinear interaction between the lower wavenumber components. Contributing factors to the amplification of the lower wavenumber modes may include the destabilizing influence of concave streamline curvature near the compression corner and the transient growth phenomenon; the relative contributions from these two factors have not yet been determined. Ongoing analysis of the curvature induced Gortler instability will shed further light on the impact of streamline curvature on the computed intensification of streaks near the compression corner.

We note that analogous, flow aligned streaks may also occur in the Purdue wind tunnel experiment [7], wherein a cylindrical roughness element is mounted along the tunnel wall. There, however, such streaks could occur not only in the wake of the roughness element but, perhaps, also within the boundary-layer flow approaching the roughness, as a consequence of the concave curvature of the upstream nozzle surface.

\section{STABILITY ANALYSIS}

As described in Section III, the mean boundary layer flow in the wake of the trip array is dominated by the presence of strong, spanwise periodic streaks that are aligned with the flow direction. Since the spanwise and wall-normal length scales of these streaks are comparable with each other, the modified boundary-layer flow has a strongly inhomogeneous character in both $y$ and $z$ directions. Therefore, its stability characteristics are more appropriately studied by solving a 2D eigenvalue problem [28-30], rather than using the conventional linear stability analysis, which is based on the assumption of basic state inhomogeneity in a single spatial coordinate (viz., the surface normal direction). Instability characteristics of streamwise streaks in low-speed boundary layers have been studied in [31-36]. Interestingly enough, findings in $[33,35,36]$ indicate that the effect of streaks can be both stabilizing or destabilizing depending on the streak amplitudes.

Spatial stability of the streaks computed for the Hyper-X configuration was examined at multiple streamwise locations in the roughness wake using the methodology described in ref. [37]. An implicit assumption behind this analysis is that any naturally occurring streaks within the boundary-layer flow are completely dominated by those induced by the trip array. For the most part, we confine our attention to "fundamental" instability modes, i.e., modes with the same spanwise wavelength as the underlying streaks. Calculations described at the end of this section indicate that the amplification rates of the most unstable subharmonic modes (which allow for discrete phase variations in fluctuating flow quantities from adjacent streaks) are bounded from the above by the growth rates of the fundamental modes of streak instability.

As is typical of the boundary-layer flows modified by finite amplitude streaks (e.g., [28-30]), multiple modes of instability tend to co-exist at a given station. The growth rates of the two dominant modes of instability at $x=7.9$ " (i.e., in the near wake of the trip array) have been plotted against the frequency parameter $\mathrm{f}$ in Fig. 8(a), which also indicates the representative mode shapes for the magnitude of the $\mathrm{u}-$ velocity perturbation associated with each mode. Despite the presence of strongly inflexional profiles in the wall-normal direction, the more unstable mode (i.e., mode 1) from abovementioned modes is found to be driven by the spanwise (z) shear of the basic state (i.e., corresponds to an odd mode according to the nomenclature of ref. [28]). Since the spanwise shear occurs solely because of the trip array, this dominant, odd (or " $z$ ") mode of streak instability would not have existed without the roughness elements. 
The subdominant mode (mode 2) is found to be an even mode that is primarily driven by the wall-normal (y) shear associated with the basic state.

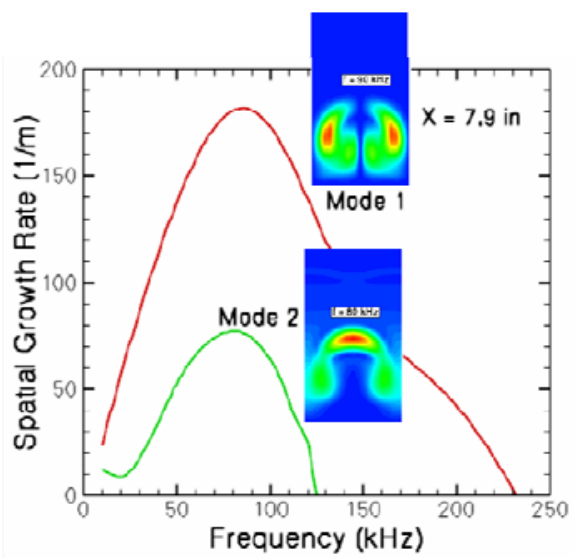

(a) Growth rates and representative modes shapes (based on the magnitude of streamwise velocity perturbation) at a fixed station $(x=7.9 ")$.

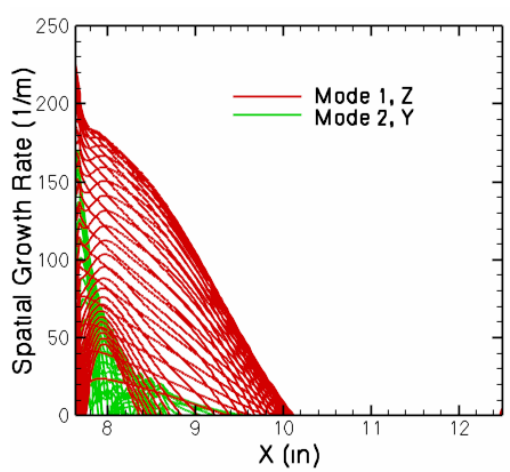

(b) Growth rates of fixedfrequency disturbances as functions of $x$. (Each curve denotes a specific frequency from the range of unstable frequencies corresponding to a given mode.)

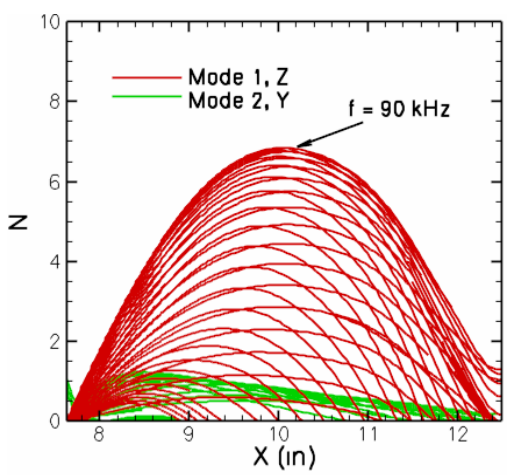

(c) Integrated logarithmic amplification ratios ( $\mathrm{N}$ factors) for fixed frequency disturbances.

Figure 8. Instability characteristics of stationary streaks due to roughness array: ramp 1 region (The $y$-z classification in parts $\mathrm{b}$ and $\mathrm{c}$ indicates the direction of basic state shear that is primarily responsible for the destabilization of a given mode.)

We next examine the spatial evolution of the above two instability modes over the first compression ramp. Streamwise variation of growth rates and cumulative amplification ratios ( $\mathrm{N}$-factors) of fixed frequency disturbances belonging to each mode has been plotted in Figs. 8(b) and 8(c), respectively. It should be noted that, because the instability analysis begins just downstream of the miniscule region of separation behind the ramp trips, the growth of instabilities in the immediate vicinity of the trip has not been accounted for in computing the $\mathrm{N}$-factors plotted in Fig. 8(c). Furthermore, the highest growth rates of mode 1 waves over the ramp 1 region occur within close proximity to the roughness array, where the influence of non-parallel effects may not be negligible because of the rapidly evolving streak amplitudes (Figs. 6(a)-(b)). Provided, however, that the distance from the trip array to the onset of transition is significantly larger than the effective length of the trip element, the stability characteristics from Figs. 8(b) and 8(c) may be suitable towards an assessment of tripping effectiveness and, perhaps, even for transition correlation.

As seen from Figs. 8(b)-(c), the growth of streak instabilities (both odd and even modes) ceases well upstream of the end of ramp 1, which is to be expected because of the rapid decrease in streak amplitudes just ahead of the compression corner (Fig. 6(b)). As seen from Fig. 8(c), the odd mode (mode 1-z) disturbances near $\mathrm{f}=90 \mathrm{kHz}$ reach a peak $\mathrm{N}$-factor of approximately 7 across an amplification region of just 2.5 inches (which, however, is more than 7 times larger than the trip length). If one assumes that $\mathrm{N}=7$ might be more than sufficient to correlate with the onset of transition in this conventional facility experiment $^{\S}$ (especially considering the potentially extra growth potential right above and slightly upstream of the trip array), then the predicted onset of transition will be within a distance of $\Delta x / L=0.05$

\footnotetext{
${ }^{\S}$ Analysis in Ref. [38], for example, indicated correlating $\mathrm{N}$ factors of less than 4 on a flared cone model in the same facility.
} 
behind the trip. This prediction is roughly consistent with the measured heating profile from Fig. 5 above, keeping in mind the uncertainty in estimating the transition onset location from the measured data (due to the difficulty in separating the increase in heat transfer due to transition from the increased laminar heating associated with the streaks). However, computations for additional cases (trip geometry or flow conditions) and/or configurations will help assess the reliability of using such correlations for roughness-induced transition in high-speed flows.

Streak instability characteristics over the second compression ramp are shown in Figs. 9(a) through (9(c), wherein we note the emergence of an additional mode of the odd (or " $z$ ") type (mode 3), which has lower amplification ratios in comparison with the first odd mode (mode 1), but is much more unstable than the even mode (mode 2). The frequency range for mode 1 unstable disturbances over the second ramp has a considerable overlap with that over the first ramp and, in fact, the disturbances with common frequencies are found (in general) to be analytic continuations of each other. In other words, those mode 1 disturbances amplify immediately behind the trip, then decay at least up to the compression corner, and begin to amplify again somewhere over the second ramp. Because the $\mathrm{N}$-factor reflects the cumulative growth of a specific disturbance entity, relative to the location where it first begins to amplify (which may be near a neutral location or immediately behind the trip array), the $\mathrm{N}$ factors for some of the mode 1 disturbances over ramp 2 have nonzero initial values (Fig. 9(c)). In contrast, modes 2 and 3 exhibit a strong decay prior to their growth over ramp 2 and, therefore, do not appear to have a meaningful connection with the unstable disturbances in the ramp 1 region.

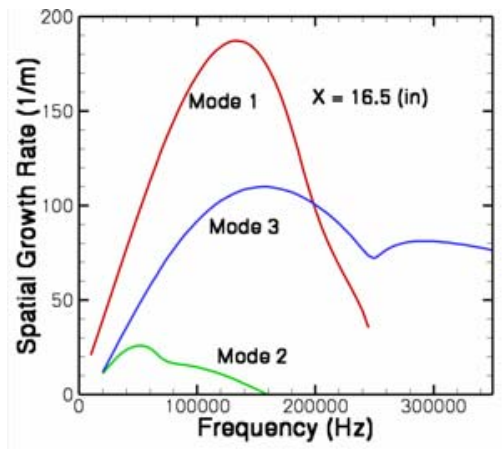

(a) Growth rates and modes shapes at $\mathrm{x}=16.5$ ", i.e., somewhat ahead of the downstream compression corner

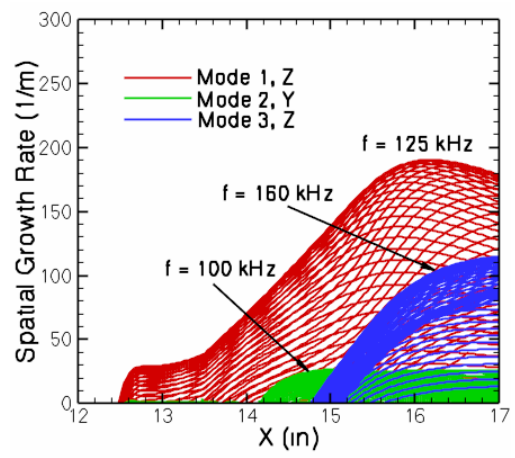

(b) Growth rates of fixed frequency disturbances as functions of $x$.

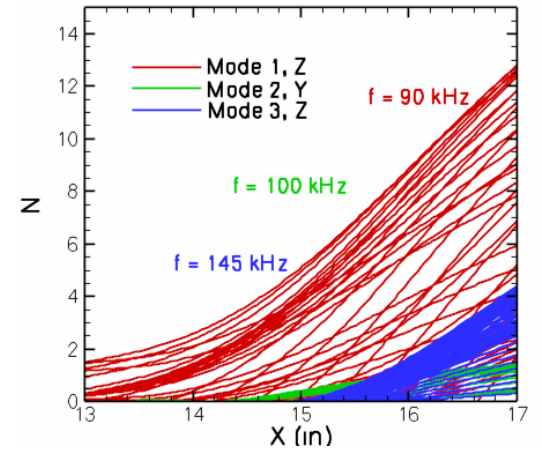

(c) Integrated logarithmic amplification ratios (i.e., $\mathrm{N}$ factors) vs. $x$.

Figure 9. Instability characteristics of stationary streaks due to roughness array: ramp 2 region

Consistent with the growth in streak amplitudes behind the first compression corner (Fig. 6(b)), the peak growth rates of streak instabilities over ramp 2 occur within the downstream portion of the ramp. While these peak growth rates are comparable to those over ramp 1, the associated $\mathrm{N}$-factors are considerably larger because of the longer region of instability. N-factor values in excess of 12 are predicted for mode 1 disturbances (with most of that growth occurring over the ramp 2 region); however, this growth might not be relevant in practice depending on the likely onset of transition over ramp 1. Because of the intervening region of strong decay in between the regions of growth on ramps 1 and 2, it is also possible to encounter somewhat abrupt variations in the transition location as the trip, flow, or facility parameters are varied.

In view of the similarity between the roughness-induced streaks and finite amplitude Gortler vortices, the structure of the odd and even modes of streak instability is analogous to that of the secondary instabilities of Gortler vortices in low-speed flows (e.g., refs. [28-30]). Of course, the substantial streamwise variation in the streak amplitudes in the present case lends additional richness to the amplification 
characteristics of the streak instabilities. Also, the sinuous modes alone appear to dominate the streak instability, whereas both sinuous and varicose modes had been found to have comparable growth rates for the incompressible Gortler vortices [30]. In Fig. 10, we present the instantaneous contours of the streamwise velocity field synthesized from a superposition of the $3 \mathrm{D}$ mean flow and the spatially growing, unsteady streak instabilities of a given type, with the initial instability amplitude assigned to be $0.1 \%$ at the origin. For simplicity, the streak flow is assumed to be parallel, and the flow visualization is synthesized on the basis of the local flow and instability characteristics at $x=7.9$. The visualizations from Figs. 10(a)-(b) confirm that the odd mode of instability induces sinuous motions of the underlying stationary streaks, whereas the even mode leads to varicose perturbations. Ref. [31] provides an excellent exposition of the manner in which the nonlinear breakdown of the sinuous and varicose modes of organized streaks leads to the onset of transition in low-speed flows.

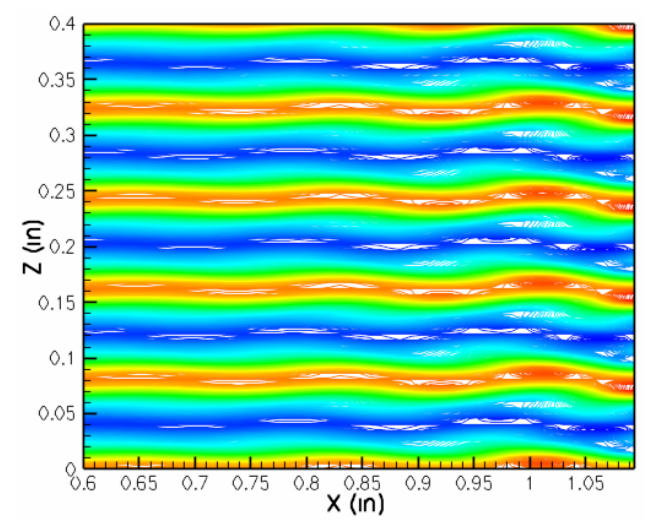

Fig. 10(a) Mode $1(\mathrm{f}=90 \mathrm{kHz})$

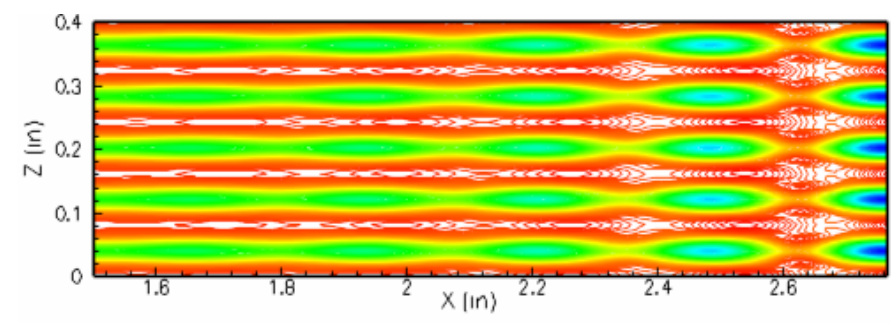

Fig. 10(b) Mode $2(\mathrm{f}=80 \mathrm{kHz})$

Figure 10. Instantaneous u-velocity contours over a suitably selected constant-y plane for the streak flow as perturbed by the instability modes 1 and 2. The abscissa in both parts of the figure denotes the distance downstream of the $\mathrm{x}=7.9$ inches location along the Hyper-X configuration.

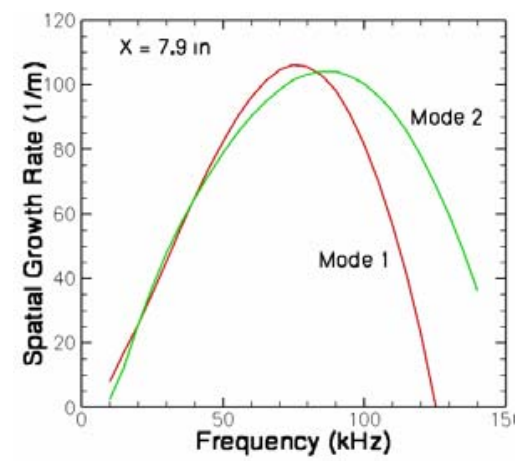

(a) Growth rates at $x=7.9$ ".

Figure 11. (Continued on next page.)

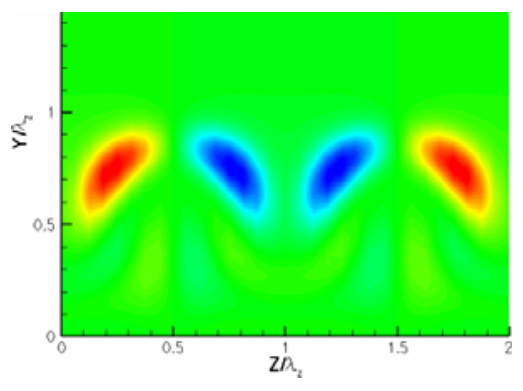

(b) Mode shape for the real part of streamwise velocity perturbation associated with the most unstable subharmonic, mode-1 disturbance at $x=7.9$ '.

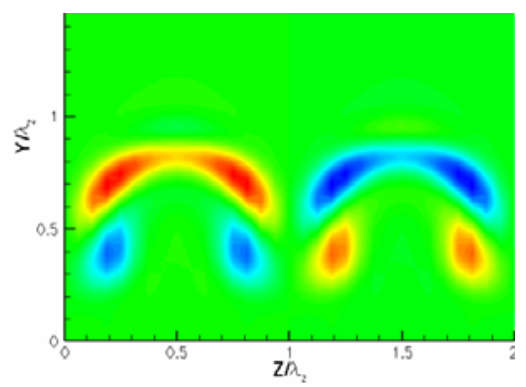

(c) Mode shape for the real part of streamwise velocity perturbation associated with the most unstable subharmonic, mode-2 disturbance at $x=7.9 "$. 


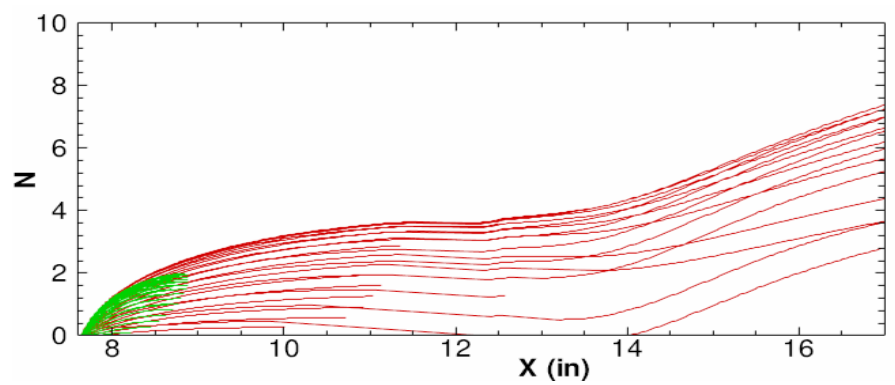

(d) Integrated logarithmic amplification ratios (i.e., $\mathrm{N}$ factors) vs. $x$.

Figure 11. Instability characteristics of subharmonic modes with a spanwise wavelength of $2 \delta$

Recall from the beginning of this section that, in addition to the fundamental modes investigated thus far, the streaks can support subharmonic modes of instability with spanwise wavelengths that are larger than the array spacing. Figure 11(a) indicates the growth rate behavior at $x=7.9$ " for a pair of subharmonic modes, each with a spanwise wavelength of $2 \delta$ (i.e., the first subharmonic of the fundamental modes). The mode shapes for these two subharmonic modes resemble the mode shapes for the fundamental modes of sinuous and varicose type, respectively, except that the fluctuations over an adjacent pair of streaks have a 180 deg phase shift between them. To emphasize that phase shift, the mode shapes in Figs. 11(b) and 11(c) reflect the real part (rather than the absolute value) of the streamwise velocity fluctuation associated with the subharmonic mode. The corresponding contours of the instantaneous streamwise velocity distribution across a suitably chosen constant-y plane are shown in Figs. 12(a) and 12(b), respectively. Interestingly enough, the peak growth rates of both subharmonic modes from Fig. 11(a) are greater in comparison with the even (varicose) fundamental mode at $x=7.9$ " (i.e., mode 2 from Fig. 8(a)). Of course, the odd (sinuous) fundamental mode (i.e., mode 1 from Fig. 8(a)) still retains its identity as the most unstable mode at this station (and, quite possibly, throughout the region of growth over ramp 1).

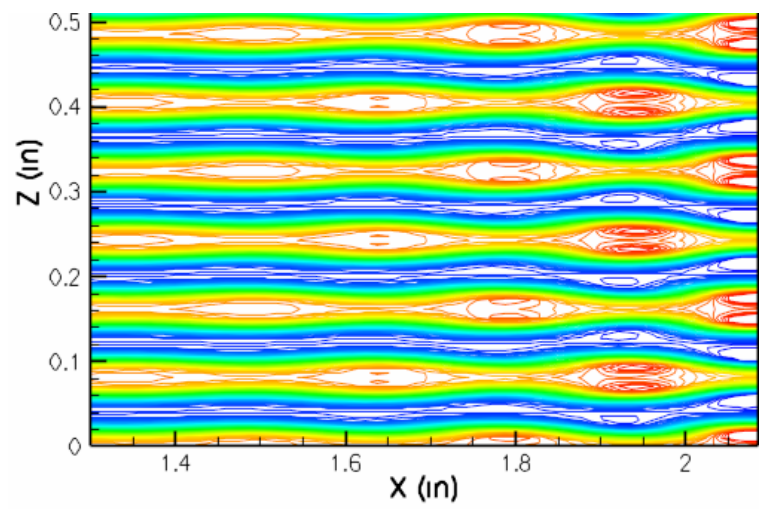

Fig. 12(a) Most unstable subharmonic, mode-1 disturbance based on local flow near $x=7.9$ ".

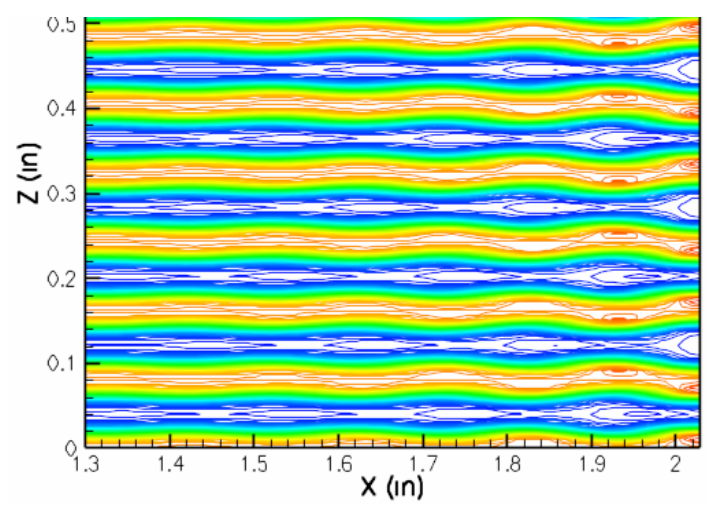

Fig. 12(b) Most unstable subharmonic, mode-2 disturbance near $x=7.9$ ".

Figure 12. Instantaneous u-velocity contours for the streak flow perturbed by the subharmonic instability modes 1 and 2. (Procedure for flow-field synthesis identical to Fig. 10.) 


\section{OTHER TRIP AND FLOW CONFIGURATIONS}

Computations were also performed for an array of diamond trips (Trip 1 from Ref. [4]) with the same height as the ramp trips ( $\mathrm{h}=0.06$ inches) examined in Sections III and IV, except with twice the spanwise array spacing (i.e., $2 \delta=0.162$ inches) (Fig. 13). Oil flow visualizations in the experiment [4] had indicated two spanwise regions of oil accumulation behind each diamond shaped trip element, one behind the trailing corner of the diamond and the other along the centerline of the gap between each pair of adjacent trip elements. This behavior was also seen in the computations, which indicated a convergence of skin-friction lines along both symmetry lines. However, convergence towards the gap centerline becomes weaker over the downstream portion of ramp 1 and is virtually absent behind this corner. Computations also indicate that the velocity amplitude of the streaks behind the diamond trips is significantly larger than that behind the ramp trips of identical height. The presence of stronger streaks behind the diamond trips was also noted in the course of experimental observations in Ref. [4]. Despite the larger streak amplitudes, however, the peak disturbance $\mathrm{N}$-factors over the first compression ramp were somewhat smaller in the case of diamond trips, in comparison with those presented earlier for the ramp trips (Fig. 8(c)). Because the same mode of instability (namely, the odd or the z-mode) dominates in both cases, the slightly lower $\mathrm{N}$-factors for the diamond trip may have been caused by the weaker spanwise gradients associated with the basic state (resulting from the larger spanwise array spacing in that case).

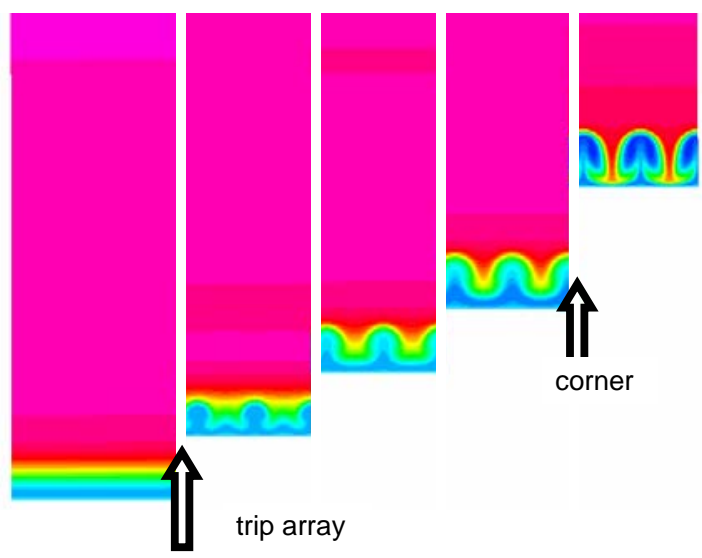

Fig. 13(a) Cross-sectional view of streaks behind diamond trips

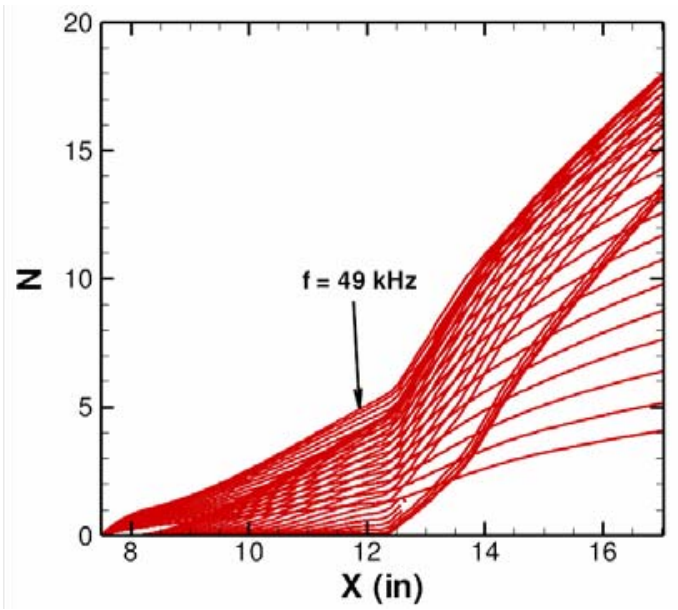

Fig. 13(b) Integrated logarithmic amplification ratios ( $\mathrm{N}$ factors) of $z$-mode disturbances vs. $x$.

Fig. 13. Spanwise periodic array of diamond trips on the Hyper-X configuration (location and height of trips same as ramp trips in Section IV).

To further understand the effect of the compression corner on streak amplitudes and resulting instability characteristics, as well as to contrast the structure of the trip wakes behind an isolated trip element with that behind a spanwise periodic trip array, computations were performed for isolated diamond trips mounted on a flat plate at zero incidence to the incoming free stream (Fig. 14). The flow conditions for these computations $\left(\mathrm{M}=3.5, \mathrm{Re}=3 \times 10^{6} / \mathrm{ft}, \mathrm{T}=90.2 \mathrm{deg} \mathrm{K}\right.$, width of each face of diamond trip $=0.05$ inches) were chosen to be relevant to a planned experiment in the Supersonic Low Disturbance Tunnel at NASA Langley Research Center [40]. 


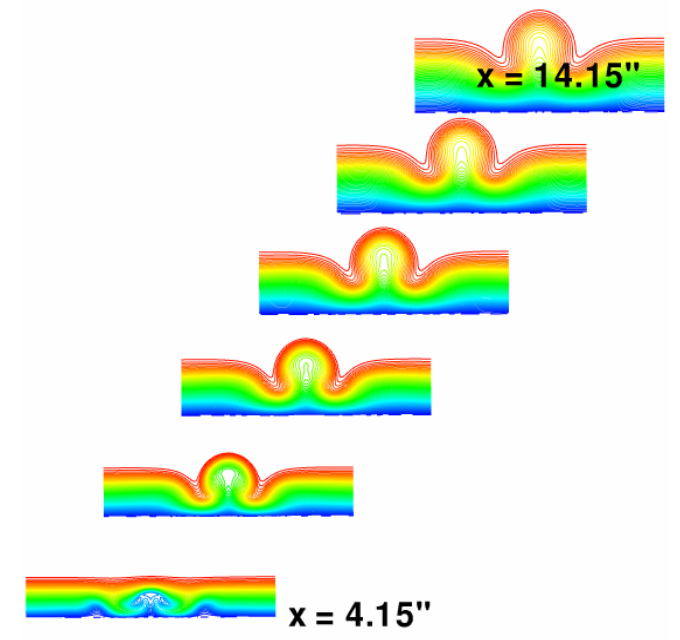

Fig. 14. Streamwise velocity contours behind an isolated, diamond trip of height 0.02 inches $\left(\mathrm{h} / \delta^{*}\right.$ $\approx 0.73$, where $\delta^{*}$ denotes the displacement thickness of the boundary layer) in a Mach 3.5 flat plate boundary layer.

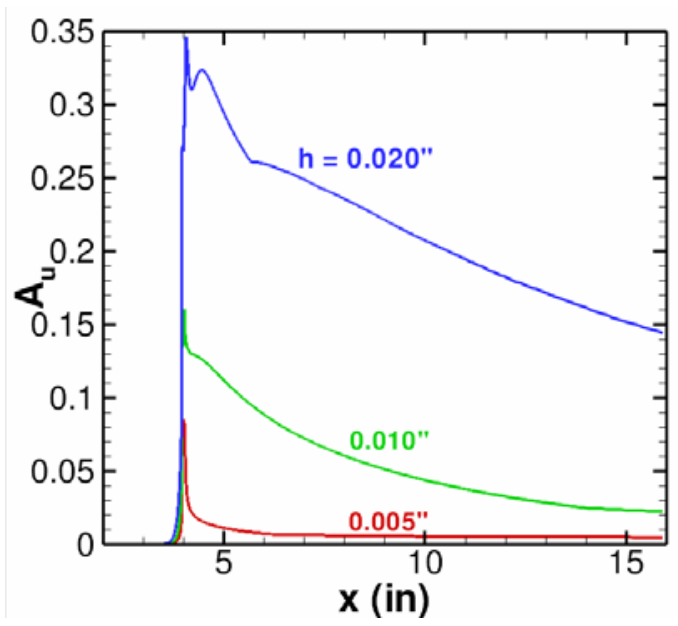

Fig. 15. Effect of trip height on streak amplitudes behind an isolated, diamond shaped trip element in Mach 3.5 boundary layer.

As shown in Fig. 15, streak amplitudes for the flat plate case decay nearly monotonically with distance from the trip (or the trip array), in contrast to the case of Hyper-X like compression surfaces where the rapid increase in streak strength near the compression corner helped sustain the streak amplitude over rather large distances. Computations also confirmed that the streak amplitudes have a strongly nonlinear dependence on the roughness height (Fig. 15), not unlike the case of roughness-induced streaks in lowspeed flows $[40,36]$. More in-depth analysis of these solutions will be deferred to a separate paper.

\section{SUMMARY}

The immersed boundary method developed by Choi et al. [20] and Edwards [21] has been used to model the flow behind a spanwise periodic array of 0.060 " tall boundary-layer trips mounted on a 0.333 scale model of the Hyper-X flight configuration. Having determined that the region of flow separation in the immediate vicinity of the roughness array is rather small (and, therefore, unlikely to cause an absolute instability), we analyzed the spatial stability of the mean boundary-layer flow in the wake of the trip array, which is strongly inhomogeneous in both surface-normal and spanwise directions. Given the rich spectrum of instability modes in this flow, which includes the Rayleigh and Gortler modes of instability in the upstream/unperturbed boundary layer and the instability modes of the boundary-layer streaks induced by the trip array, transition in the presence of the trips may involve an intricate mixture of instability modes. Nonetheless, the initial findings are encouraging in suggesting that, for a range of intermediate trip heights between the critical and effective values, the streak instability may play a dominant role during the transition process. Similar streaks also occur behind isolated roughness elements and it will be interesting to assess their role during the transition process, especially in the absence of any compression corner along the surface (since the corner tends to strengthen the streak amplitudes as noted in the present case).

The stability analysis predicts strong amplification of sinuous instability modes associated with the spanwise gradients of stationary streaks within the array wake, with the cumulative disturbance amplification approaching $\mathrm{N}=7$ over a distance of approximately 2.5 inches behind the trip location. Thus, even though substantially stronger instability amplification is predicted to occur over the second ramp (i.e., between the first and second compression corners), theory suggests that transition at the trip 
height and spanwise spacing of 0.060 " and 0.081 ", respectively, is likely to occur over the first ramp itself. Additional calculations are, however, necessary to determine whether or this prediction will also hold for the trip spacing of 0.12 " in the experiment [4].

It is significant to note an additional implication of the stability characteristics encountered for the ramp trip with a width of $\mathrm{h}=0.06$ inches. Following an initial region of rapid growth ahead of the first compression corner, the fundamental mode of streak instability becomes weaker over an intermediate region near this corner. Thus, it is conceivable that, if and when the amplification ahead of the corner is not sufficient to induce the onset of transition (due to low levels of facility disturbances, for example), the transition location will shift to the second region of growth, which is significantly farther downstream from the corner. In cases such as this, one might observe somewhat abrupt variations in the transition location over non-isentropic compression surfaces when the flow conditions, roughness parameters, or the disturbance environment are varied. The question of whether or not a similar variation encountered in refs. $[4,41]$ can be explained along these lines remains to be seen.

Additional computations for the diamond trip configuration have provided further insights into the behavior of flow past boundary layer trips, at least for one additional subclass of relevant trip geometries as mounted on both a flat plate and a compression surface.

We close this paper by reiterating on certain aspects of the stability analysis for stationary roughness wakes that were not addressed herein, but should be worthwhile to examine during future studies. For the predictions presented herein, the region of upstream extent was small and, therefore, we chose to ignore the instability growth within the upstream region. However, for transition predictions, in general, it may be necessary to include both the upstream region and the region immediately above the trip (along with non-parallel effects). The latter could also be accomplished via direct numerical simulations of the entire region, and the stability analysis presented herein will then provide the foundation for such computations.

\section{Acknowledgments}

The work of MC and FL was performed as part of the Aerodynamics, Aerothermodynamics, and Plasma Dynamics (AAP) discipline of the Hypersonics Project of NASA's Fundamental Aeronautics Program (FAP). Development, validation, and improvements to the stability analysis code were carried out under the Supersonic Cruise Efficiency - Airframe component of the Supersonics Project. Present results were originally documented in the Spring 2008 issue of the AAP Quarterly Newsletter edited by Dr. Seokkwan Yoon. The work of J.R. Edwards is funded under the AFOSR grant FA9550-0701-0191, with Dr. John Schmisseur as the Program Manager. The authors would like to thank Scott Berry of NASA Langley Research Center for clarifying a number of aspects of the Hyper-X trip experiment modeled in this paper. Useful technical discussions with Drs. Aaron Auslender, Thomas Horvath, and Mujeeb Malik of NASA Langley Research Center are also acknowledged.

\section{References}

1. Reda, D.C., "Review and Synthesis of Roughness-Dominated Transition Correlations for Reentry Applications," J. Spacecraft and Rockets, vol. 39, No. 2, March-April 2002.

2. Schneider, S.P., "Effects of Roughness on Hypersonic Boundary-Layer Transition," AIAA Paper 2007-305, 2007

3. Berry, S.A., and Horvath, T.J., "Discrete Roughness Transition for Hypersonic Flight Vehicles," AIAA Paper 2007-307, 2007.

4. Berry, S.A., Auslender, A.H., Dilley, A.D., and Calleja, J.F., "Hypersonic Boundary-Layer Trip Development for HyperX,”J. Spacecraft and Rockets, vol. 38, No. 6, pp. 853-864, Nov.-Dec. 2001.

5. Danehy, P.M., Garcia, A.P., Borg, S., Dyakonov, A.A., Berry, S.A., (Wilkes)Inman, J.A., and Alderfer, D.W., "Fluorescence Visualization of Hypersonic Flow Past Triangular and Rectangular Boundary-Layer Trips," AIAA Paper 2007-536, 2007

6. Borg, M.P., and Schneider, S.P., "Effect of Freestream Noise on Roughness-Induced Transition for the X-51A Forebody," AIAA Paper 2008-592, 2008.

7. Casper, K., Wheaton, B., Johnson, H., and Schneider, S., "Effect of Freestream Noise on Roughness-Induced Transition at Mach 6," AIAA Paper 2008-4291, 2008.

8. Berry, S.A., Chen, F.-J., Wilder, M., and Reda, D., "Boundary Layer Transition Experiments in Support of the Hypersonics Program," AIAA Paper 2007-4266, 1007. 
9. Berry, S.A., Daryabeigi, K., Wurster, K., and Bittner, R.D., “Boundary-Layer Transition on X-43A,” AIAA Paper 20083736, 2008.

10. Kimmel, R.L., Adamczak, D., Gaitonde, D., Rougeux, A., and Hayes, J.R., "HIFiRE-1 Boundary Layer Transition Experiment Design," AIAA Paper 2007-534, 2007.

11. Campbell, C.H., Garske, M., Kinder, J., and Berry, S.A., "Orbiter Entry Boundary Layer Flight Testing," AIAA Paper 2008-635, 2008.

12. Horvath, T.J., Berry, S.A., Schwarz, R., Daryabeigi, K., Splinter, S., and Ross, M., “Assessment and Mission Planning Capability for Quantitative Aerothermodynamic Flight Measurements Using Remote Imaging," AIAA Paper 2008-4022, 2008.

13. Marxen, O., Iaccarino, G., and Shaqfeh, E.S.G., "Numerical Simulation of the Effect of a Roughness Element on HighSpeed Boundary-Layer Instability," AIAA Paper 2008-4400, 2008.

14. Greene, P., "A Numerical Study of Purdue's Mach 6 Tunnel with a Roughness Element," AIAA Paper 2009-0174, 2009.

15. Chang, C.-L., and Choudhari, M., "Hypersonic Viscous Flow over Large Roughness Elements," AIAA Paper 2009-0173, 2009.

16. Van Driest, E. R., and McCauley, W.D., "The Effect of Controlled Three-Dimensional Roughness on Boundary-Layer Transition at Supersonic Speeds,” J. Aero. Sc., vol. 27, No. 4, pp. 261-271, 1960.

17. Acarlar, M.S., and Smith, C.R., "A Study of Hairpin Vortices in a Laminar Boundary Layer. Part 1." J. Fluid Mech., Vol. 175, pp. 1-42, 1987.

18. Andersson, P., Berggren, M., and Henningson, D.S., "Optimal Disturbances and Bypass Transition in Boundary Layers," Phys. Fluids, Vol. 11(1), pp. 134-150, 1999.

19. Reshotko, E. and Tumin, A., "Investigation of the Role of Transient Growth in Roughness-Induced Transition," AIAA Paper 2002-2850, 2002.

20. Colella, P., and Woodward, P.R., "The Piecewise Parabolic Method (PPM) for Gas- Dynamical Simulations, J. Comp. Phys., Vol. 54, pp. 174-201, 1984.

21. J.-I. Choi, R.C. Oberoi, J.R. Edwards, and J.A. Rosati, An Immersed Boundary Method for Complex Incompressible Flows, J. of Comp. Physics, Vol. 224, pp. 757-784, 2007.

22. Edwards, J.R., "Hybrid LES/RANS Simulation of the Effects of Boundary Layer Control Devices Using Immersed Boundary Methods," Interim Report for AFOSR Grant FA9550-0701-0191, 2008.

23. Korolev, G.L., Gajjar, J.S.B., and Ruban, A.I., "Once Again on Supersonic Flow Separation near a Corner," J. Fluid Mech., vol. 463, pp. 173-199, 2002

24. Balakumar, P., Zhao, H., and Atkins., H., "Stability of Hypersonic Boundary Layers over a Compression Corner," AIAA Paper 2002-2848, 2002.

25. Fletcher, A.I.P., Ruban, A.I., and Walker, J.D.A., "Instabilities in Supersonic Compression Ramp Flow," J. Fluid Mech., vol. 517, pp. 309-330.

26. Gaitonde, D.V., Canupp, P.W., and Holden, M.S., "Heat Transfer Predictions in a Laminar Hypersonic Viscous/Inviscid Interaction," J. Thermophysics and Heat Transfer, vol. 16, No. 4, pp. 481-489, 2002.

27. Druguet, M.-C., Candler, G.V., and Nompelis, I., "Simulations of Viscous Hypersonic Double-Cone Flows: Influence of Numerics," AIAA Paper 2003-3548, 2003.

28. Hall, P. \& Horseman, N.J. 1991 The linear inviscid secondary instability of longitudinal vortex structures in boundary layers. J. Fluid Mech. 232, 357-375.

29. Yu, X., and Liu, J.T.C., "On the Mechanism of Sinuous and Varicose Modes in Three-Dimensional Viscous Secondary Instability of Nonlinear Gortler Rolls," Phys. Fluids, vol. , pp. 736-750, 1994.

30. Li, F., and Malik, M.R., "Fundamental and Subharmonic Secondary Instability of Gortler Vortices," J. Fluid Mech., vol. 297, pp. 77-100, 1995.

31. Brandt, L., and Henningson, D.S., "Transition of Streamwise Streaks in Zero-Pressure-Gradient Boundary Layers," J. Fluid Mech., vol. 472, pp. 229-261, 2002.

32. Goldstein, M.E., and Wundrow, D.W., "Interaction of Oblique Instability Waves with Weak Streamwise Vortices," J. Fluid Mech., vol. 284, p. 277-307, 1995.

33. Cossu, C., and Brandt, L., "Stabilization of Tollmien-Schlichting Waves by Finite Amplitude Optimal Streaks in the Blasius Boundary Layer," Phys. Fluids, Vol. 14, L57-L60, 2002.

34. Wu, X., and Luo, J., "Linear and Nonlinear Instabilities of a Blasius Boundary Layer Perturbed by Streamwise Vortices. Part 1. Steady Streaks," J. Fluid Mech., Vol. 483, pp. 225-248, 2003.

35. Fransson, J.H.M., Brandt, L., Talamelli, A., and Cossu, C., "Experimental Study of the Stabilization of TollmienSchlichting Waves by Finite Amplitude Streaks," Proceedings of IUTAM Symposium on Laminar-Turbulent Transition, Bangalore, India, Dec. 13-17, 2004.

36. Choudhari, M. and Fischer, P., "Roughness-Induced Transient Growth" AIAA Paper 2005-4765, 2005.

37. Li, F., and Choudhari, M., "Spatially Developing Secondary Instabilities and Attachment Line Instability in Supersonic Boundary Layers," AIAA Paper 2008-590, 2008.

38. Horvath, T. J., Berry, S. A., Hollis, B. R., Chang, C.-L., and Singer, B. A., "Boundary Layer Transition On Slender Cones In Conventional and Low Disturbance Mach 6 Wind Tunnels," AIAA Paper 2002-2743, 2002.

39. Kegerise, M., Private communication, 2008. 
40. White, E.B., and Ergin, F.G., "Receptivity and Transient Growth of Roughness-Induced Disturbances," AIAA Paper 2003-4243, 2003.

41. Berry, S.A., Nowak, R., and Horvath,, T.J., "Boundary Layer Control for Hypersonic Airbreathing Vehicles," AIAA Paper 2004-2246, 2004. 